\title{
The case for evidence-based medicine for the association between hyperuricaemia and CKD
}

\author{
Stefanie Steiger (D), Qiuyue Ma and Hans-Joachim Anders (iD)
}

We write in response to the Perspectives article by Y. Sato et al. (The case for uric acid-lowering treatment in patients with hyperuricaemia and CKD. Nat. Rev. Nephrol. 15, 767-775; 2019) ${ }^{1}$, which advocated treating asymptomatic hyperuricaemia to attenuate the progression of chronic kidney disease (CKD), based on small randomized control trials (RCTs) categorized as either 'interpretable' or 'non-interpretable'. The majority of interpretable RCTs suggested a treatment effect, whereas non-interpretable RCTs did not. The authors concluded that "treatment of so-called asymptomatic hyperuricaemia to slow or delay the progression of CKD should be a key management strategy" . We have several objections to this statement.

First, 5 of 14 'interpretable' studies that reported positive results were not retrievable via Pubmed, Medline or Google Scholar and were only available from one Chinese repository of research literature. Q.M., who is a native Chinese speaker, analysed these publications and found variations between the full text and the English abstract - for example, urate-lowering therapy (ULT) with benzbromarone was mentioned in the English abstract but not in the full text of one study ${ }^{2}$, which might lead to misinterpretation of the study. By contrast, a post-hoc analysis of two replicate phase III RCTs that was published open access in English and fulfilled the interpretable criteria established by the authors but reported negative results ${ }^{3}$ was omitted from their analysis ${ }^{1}$, suggesting a selection bias towards the concept proposed by the authors.

Second, the analysis by the authors is flawed because the threshold criteria change in estimated glomerular filtration rate (eGFR) and/or change in creatinine clearance in controls, which were used to identify interpretable studies, were not corrected in the control group for trial length (range 1-84 months). This normalization for trial length should be a mandatory criterion when assessing whether a study of CKD progression is interpretable. Moreover, the authors included trial outcome as a selection criterion for classifying a study as interpretable, which implies a self-fulfilling prophecy ${ }^{4}$.
Third, 9 of 14 'interpretable' RCTs were either underpowered, reported an improvement in eGFR in the control arm, were single-centre RCTs and/or were not registered in clinicaltrials.gov before publication. In our opinion, collectively, these studies do not provide sufficient evidence for changes in treatment recommendations ${ }^{5}$; regulatory authorities only consider well-powered multi-centre $\mathrm{RCTs}^{4}$ for this purpose.

Since publication of the article by Sato et al., preliminary results from two large multicentre RCTs have been reported ${ }^{6,7}$ and consistently failed to show a causal link between asymptomatic hyperuricaemia and CKD progression (Supplementary information). Furthermore, as acknowledged by the authors ${ }^{1}$, two large Mendelian randomization studies ${ }^{8,9}$ had shown no significant causal relationship between serum uric acid level and risk of CKD.

After revising the analysis according to the aforementioned points, a meta-analysis of all single-centre RCTs suggests a small treatment effect size of $10 \%$ (overall odds ratio (OR) 0.90). By contrast, a meta-analysis of multi-centre RCTs demonstrates that ULT does not attenuate CKD progression (overall OR 1.0) (Supplementary information). In the case of asymptomatic hyperuricaemia and CKD, the best available scientific evidence does not support the use of ULT in patients with asymptomatic hyperuricaemia and CKD, at least not with the purpose of attenuating CKD progression.

There is a reply to this letter by Sato, Y. et al. Nat. Rev. Nephrol. https://doi.org/10.1038/ s41581-020-0289-2 (2020)

\section{Stefanie Steiger (ID), Qiuyue Ma and Hans-Joachim Anders $\mathbb{D}$ 凶 \\ Department of Medicine IV, University Hospital, LMU Munich, Munich, Germany. 凶e-mail: hjanders@med.uni-muenchen.de https://doi.org/10.1038/s41581-020-0288-3}

1. Sato, Y. et al. The case for uric acid-lowering treatment in patients with hyperuricaemia and CKD. Nat. Rev. Nephrol. 15, 767-775 (2019).

2. Tan, Y., Fu, J., Liang, M., Lin, Z. \& Huang, J. Clinical observation of the effect of allopurinol to protect renal function in patients with diabetic nephropathy. Mod. Hosp. 11, 36-38 (2011)

3. Yood, R. A., Ottery, F. D., Irish, W. \& Wolfson, M. Effect of pegloticase on renal function in patients with chronic kidney disease: a post hoc subgroup analysis of 2 randomized, placebo-controlled, phase 3 clinical trials. BMC Res. Notes 7, 54 (2014).

4. Downing, N. S., Aminawung, J. A., Shah, N. D., Krumholz, H. M. \& Ross, J. S. Clinical trial evidence supporting FDA approval of novel therapeutic agents, 2005-2012. JAMA 311, 368-377 (2014).

5. Djulbegovic, B. \& Guyatt, G. H. Progress in evidencebased medicine: a quarter century on. Lancet 390 , 415-423 (2017).

6. Badve, S. et al. Effect of allopurinol on the progression of CKD: the CKD-FIX study[abstract TH-PO1198] ASN Kidney Week https://www.asn-online.org/ education/kidneyweek/2019/program-abstract. aspx? controlld=3261360 (2019).

Doria, A., Gelecki, A., Spino, C. \& Maurer, M. Preventing Early Renal Loss in Diabetes (PERL) study: outcome of a 3-year trial of serum uric acid reduction with allopurinol [abstract FR-OR137] ASN Kidney Week https:/www.asn-online.org/education//kidneyweek/2019/ program-abstract.aspx? controlld=3274268 (2019).

8. Ahola, A. J. et al. The serum uric acid concentration is not causally linked to diabetic nephropathy in type 1 diabetes. Kidney Int. 91, 1178-1185 (2017).

9. Jordan, D. M. et al. No causal effects of serum urate levels on the risk of chronic kidney disease: a

Mendelian randomization study. PLoS Med. 16 e1002725 (2019).

\section{Acknowledgements}

This work was supported by the Deutsche Forschungsgemeinschaft (STE2437/2-1 and AN372/24-1) and by LMUexcellent initiative (S.S.). Q.M. was supported by the Chinese Scholarship Council.

\section{Competing interests}

The authors declare no competing interests.

Supplementary information

Supplementary information is available for this paper at https://doi.org/10.1038/s41581-020-0288-3.

\section{Reply to 'The case for evidence-based medicine for the association between hyperuricaemia and CKD'}

Yuka Sato, Daniel I. Feig, Austin G. Stack, Duk-Hee Kang, Miguel A. Lanaspa,

A. Ahsan Ejaz, L. Gabriela Sánchez-Lozada, Masanari Kuwabara(D),

Claudio Borghi and Richard J. Johnson (1)

In our Perspectives article (The case for uric acid-lowering treatment in patients with hyperuricaemia and CKD. Nat. Rev. Nephrol. 15, 767-775; 2019) $)^{1}$ we proposed that individuals with hyperuricaemia and chronic kidney disease (CKD) with worsening kidney function should be considered for urate-lowering therapy (ULT) to 